\title{
Mecanismo de Desenvolvimento Limpo Florestal e a Heveicultura no Estado do Acre
}

\author{
Aldione da Silva Lessa ${ }^{1}$, Romano Timofeiczyk Junior ${ }^{2}$, \\ Zenobio Abel Gouvea Perelli da Gama e Silva², \\ Anadalvo Santos Juazeiro dos Santos ${ }^{2}$, Vitor Afonso Hoeflich ${ }^{2}$
}

\begin{abstract}
${ }^{1}$ Serviço Brasileiro de Apoio às Micro e Pequenas Empresas - SEBRAE-Acre, Rio Branco/AC, Brasil ${ }^{2}$ Departamento de Economia Rural e Extensão, Universidade Federal do Paraná - UFPR, Curitiba/PR, Brasil ${ }^{3}$ Centro de Ciências Biológicas e da Natureza, Universidade Federal do Acre - UFAC, Rio Branco/AC, Brasil
\end{abstract}

\section{RESUMO}

O objetivo deste artigo foi gerar informações econômicas sobre plantios de seringueira (Hevea spp.) inseridos no mercado de carbono no âmbito do mecanismo de desenvolvimento limpo (MDL) e implantados no estado do Acre. Para tal, considerando dados sobre os projetos locais de seringal de cultivo, foram quantificados o Valor Presente Líquido (VPL) e o Valor Esperado da Terra (VET), assim como a Taxa Interna de Retorno (TIR) desses plantios. Dos resultados gerados, concluiu-se que os reflorestamentos com seringueiras destinados ao sequestro de carbono no âmbito do MDL Florestal, apresentam potencial para serem viáveis, caso implantados no Acre e, assim, fortalecerem a economia de base florestal dessa região, desde que a sua renda não sofra uma queda de $20 \%$, ou mais, em relação ao valor médio obtido neste estudo.

Palavras-chave: mudanças climáticas, seringueira (Hevea spp.), Amazônia.

\section{Forestry Clean Development Mechanism and the Heveicuture in the State of Acre, Brazil}

\begin{abstract}
The objective of this paper is to generate economic information on rubber tree (Hevea spp.) plantations included in the carbon market under the clean development mechanism (CDM), deployed in the state of Acre, Brazil. To this end, considering data from local heveiculture projects, Net Present Value (NPV), Land Expectation Value (LEV), and Internal Rate of Return (IRR) were quantified for theses reforestations. Based on the results obtained, we conclude that reforestation with rubber trees for carbon sequestration under Forestry CDM may be feasible in the state of Acre, thus strengthening the forest-based economy of the region, provided that the income generated from these plantations is not reduced by more than $20 \%$ compared with the average value obtained at the time of this study.
\end{abstract}

Keywords: climate change, rubber tree (Hevea spp.), Amazonia. 


\section{INTRODUÇÃO}

UNFCCC (2003) define mudança climática como [...] qualquer alteração que possa ser, diretamente ou indiretamente, atribuída à atividade humana que modifique a composição da atmosfera e que se some àquela provocada pela variabilidade climática natural observada ao longo de períodos comparáveis.

IPCC (2007) acrescenta que alterações nos regimes de chuva e ventos, derretimento das calotas polares, aumento do nível dos oceanos, inundações de cidades litorâneas, eventos climáticos extremos e acirramento dos processos de desertificação advêm do aquecimento global. Fernandes (2003), por sua vez, afirma que tal elevação da temperatura se deve às altas concentrações de gases de efeito estufa na atmosfera.

Complementando, IPCC (2007) lista, como ações humanas que mais emitem gases de efeito estufa (GEE), a geração de energia pela queima de combustíveis fósseis e o desmatamento, entre outras. Os principais gases de efeito estufa são Dióxido de carbono $\left(\mathrm{CO}_{2}\right)$, Metano $\left(\mathrm{CH}_{4}\right)$, Óxido nitroso $\left(\mathrm{N}_{2} \mathrm{O}\right)$, Perfluorocarbonos (PFC), Hidrofluorocarbonos (HFC) e Hexafluoreto de enxofre $\left(\mathrm{SF}_{6}\right)$.

No tema mudanças climáticas, Brasil (2012) destaca o Protocolo de Quioto como o principal documento elaborado, até hoje, pela cúpula internacional sobre mudanças antropogênicas na temperatura do planeta. Esse protocolo, criado em 1997 e ratificado em 2005, obrigava os países industrializados a reduzirem, entre 2008 e 2012, suas emissões combinadas de gases de efeito estufa em no mínimo 5\% em relação ao nível de 1990 para assim reverter a tendência histórica de crescimento das emissões nesses países.

Stern (2006) argumenta que para limitar a mudança do clima a um aumento da concentração de 550 ppm em volume até o ano 2050 será movimentado algo como $1 \%$ do Produto Interno Bruto (PIB) mundial até essa data. CGEE (2010) ressalta que, ao assinarem a United Nations Framework Convention on Climate Change (UNFCCC), as nações aceitaram elaborar uma estratégia global para combater a poluição atmosférica. Com isso, demostraram que reconhecem a mudança do clima como uma preocupação da humanidade.

Fernandes (2003) e Cotta et al. (2008) defendem que o sequestro de carbono via atividades florestais baseia-se em duas premissas: a) os efeitos dos esforços para remover os GEE independem de sua área de aplicação, se próximos à fonte ou do outro lado do mundo, já a vegetação verde, via fotossíntese, tira o gás carbônico da atmosfera, usando-o para gerar açúcar e outros compostos orgânicos empregados no seu crescimento e metabolismo; e b) as plantas lenhosas armazenam carbono na madeira e em outros tecidos até a sua morte e decomposição, quando o carbono na madeira pode ser desprendido como gás carbônico, monóxido de carbono, metano ou mesmo incorporado ao solo como substância orgânica.

O documento sobre Redução Certificada de Emissões (RCEs) em florestamento e reflorestamento pode ser de caráter temporário, caso expire no final do período de compromisso subsequente ao que foi emitido, ou de longo prazo, se ele se encerra ao final do período de creditação para o qual foi emitido (CGEE, 2010). Já para Walter \& Coltri (2009), o período de creditação, em função das espécies usadas, finalidade do plantio e possível colheita ou não da madeira, pode ser de 20 anos, passíveis de renovação por duas vezes, ou de 30 anos sem renovação. Além disso, os projetos com remoções antrópicas líquidas de GEEs menores que $16.000 \mathrm{tCO}_{2}$ eq. (dióxido de carbono equivalente) por ano são de pequena escala, enquanto os sem limites de tamanho ou de remoção líquida são de grande escala.

Salienta-se que o estado do Acre tem a sua relevância nos esforços para controlar o aquecimento global, pois como informam Forest Trends \& The Katoomba Group (2011), em 2010 foi criado nesse estado o programa Sistema Estadual de Incentivos a Serviços Ambientais (SISA-Carbono). Tal programa, executado pelas secretarias e autarquias do governo local, integradas com prefeituras e movimentos sociais organizados, consolida a política de valoração do ativo ambiental florestal acriano.

Por seu turno, a heveicultura, segundo Nishi (2003), é uma opção viável para o MDL Florestal, pois essa atividade florestal tem um elevado potencial de sequestro de carbono, com aumento de rendimento da propriedade, fixa o homem à terra e, sendo uma cultura ajustada às áreas degradadas, promove sua estabilização e recuperação.

Cabe aqui enfatizar que a análise econômica é um fator-chave para justificar a implantação de projetos no âmbito do MDL Florestal. Assim, Berger et al. (2011) apontam como principais critérios adotados na avaliação da economicidade de projetos florestais 
o Valor Presente Líquido (VPL), a Taxa Interna de Retorno (TIR) e o Valor Esperado da Terra (VET).

Nesse contexto, Nishi et al. (2005), Silva \& Fontes (2005) e Valdetaro et al. (2011) citam que o VPL de um projeto é obtido subtraindo-se o valor presente dos custos do valor presente das receitas. Assim sendo, o projeto será considerado viável, economicamente, caso o seu valor presente líquido seja positivo.

Já a TIR, conforme mencionam Sanguino et al. (2007) e Valdetaro et al. (2011), é a taxa de desconto que iguala o valor presente das receitas ao valor presente dos custos. Conforme esse critério, o projeto será viável se a sua taxa interna de retorno for maior que uma taxa mínima de atratividade.

Complementando, Berger et al. (2011) definem o Valor Esperado da Terra como a técnica que indica o valor máximo que se pode pagar pela terra para que o projeto seja remunerado numa determinada taxa de juro. Assim, esse valor subsidia as tomadas de decisões de investidores e as formulações de políticas de governo ligadas ao setor florestal.

Diante do exposto, este artigo tem como objetivo maior gerar informações econômicas sobre o plantio de seringueira inserido no mercado de carbono no âmbito de MDL implantados no estado do Acre. Em termos específicos, este estudo buscou analisar a economicidade da heveicultura no âmbito do MDL Florestal nessa região.

\section{MATERIAL E MÉTODOS}

\subsection{Material}

\subsection{1. Área de estudo}

O cenário escolhido para este estudo foi o estado do Acre que, como relatado por Acre (2011), situa-se no norte do Brasil e, pela classificação de Köppen, tem um clima do tipo equatorial, quente e úmido. A temperatura média anual varia de $24,5{ }^{\circ} \mathrm{C}$ a $32{ }^{\circ} \mathrm{C}$, é uniforme em todo o estado e predomina em toda a região amazônica.

Complementando, o IBGE (2010) cita que o estado do Acre tem uma área total de $164.123 \mathrm{~km}^{2}$ e que $12 \%$ dela é desmatada. No estado, a umidade relativa do ar atinge $90 \%$, com o índice pluviométrico variando de $1.600 \mathrm{~mm}$ a $2.750 \mathrm{~mm} /$ ano.

\subsubsection{Dados básicos}

A identificação do material a ser avaliado nesse estudo seguiu recomendações de Berger et al. (2011). Assim, foram coletados os valores de custo, produção e renda nos plantios de seringueira para inserção no MDL Florestal, os quais são descritos a seguir:

a) Preço da terra

Por sugestão de Mastrângelo (2008), considerou-se o preço da terra como sendo R $\$ 1.000 /$ ha. Com esse valor, em 2007, comprava-se a terra, sem a cobertura florestal nativa, num raio de $80 \mathrm{~km}$ da capital Rio Branco, com acesso rodoviário o ano todo.

b) Preço e produtividade do látex

O preço do látex coagulado, usado nessa análise econômica, era de R \$3.500/t, ou US\$1,88/kg. Esse valor, praticado em 2012, foi coletado em uma visita à Secretaria de Estado de Extensão Agroflorestal e Produção Familiar do Estado do Acre (SEAPROF).

Já, a produtividade do látex coagulado, nos seringais de cultivo no Acre, é de $600 \mathrm{~kg} / \mathrm{ha} /$ ano no seu primeiro ano de produção (aos 7 anos de idade do plantio). Esse valor vai aumentando até o $15^{\circ}$ ano, quando estabiliza em $2.000 \mathrm{~kg} / \mathrm{ha} / \mathrm{ano}$, até o final do ciclo de produção. Tais dados foram obtidos em visita à Secretaria de Estado de Extensão Agroflorestal e Produção Familiar do Estado do Acre (SEAPROF).

c) Custos de implantação e manutenção de projetos com Hevea spp.

Para compor os custos dos plantios com Hevea spp. no estado do Acre, conforme indicado na Tabela 1, fez-se uma adaptação dos dados levantados na Secretaria de Estado de Extensão Agroflorestal e Produção Familiar do Estado do Acre (SEAPROF).

\section{d) Base de dados de biomassa/carbono}

Na identificação da base de dados sobre biomassa/ carbono foram usadas as informações relatadas por Lima (2010). Nesse estudo, o valor médio da biomassa armazenada nas árvores em todos os seus compartimentos (folhas, galhos, fustes e raízes) é de 51,73 t/ha, enquanto que o valor máximo é de 69,02 t/ha de biomassa.

Com os dados de biomassa, projetou-se a absorção líquida de carbono, considerando-se que 50\% da matéria 
Tabela 1. Custos num projeto de reflorestamento com Hevea spp., estado do Acre, 2012.

Table 1. Costs of a reforestation project with Hevea spp., state of Acre, 2012.

\section{Ano de ocorrência}

0

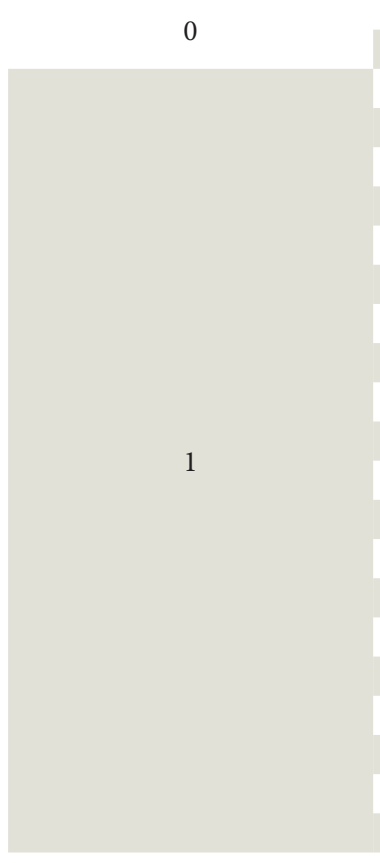

$2,3,4,5,6$

7

$$
\begin{gathered}
8,9,10,11,12,13,14,15,16, \\
17,18,19,20,21,22,23,24,
\end{gathered}
$$$$
25,26,27,28,29,30,31
$$

\section{Item/Atividade}

Valor da terra nua

Assistência técnica

Demarcação de área

Análise do solo

Destoca

Gradagem

Calcário dolomítico

Balizamento

Alinhamento e piqueteamento

Abertura e adubação de cova

Plantio

Replantio

Cobertura morta

Mudas enxertadas + (10\% mortas)

Adubo super triplo

Fungicida

Inseticida

Formicida

Pulverizador

Máscara

Bota de borracha

Boca de lobo

Pulverização

Desbrotamento

Adubação em cobertura

Roçagem

Cobertura morta

NPK 12-10-3,6

Adubação em cobertura

Roçagem

NPK 12-10-3,6

Suporte de arame

Bica

Tigela

Faca

Abertura painel

Sangria

Adubação em cobertura

Roçagem

NPK 12-10-3,6

Mão de obra

\section{US\$/hectare}

615,91

656,32

26,88

26,88

274,19

172,04

14,92

26,88

53,76

80,65

53,76

13,44

26,88

$1.687,10$

48,17

91,40

48,39

23,66

126,88

59,14

19,89

26,08

53,76

80,65

26,88

241,94

26,88

107,53

26,88

80,65

118,28

127,96

63,98

63,98

26,88

40,32

134,41

26,88

161,29

150,54

752,69

Fonte: dados coletados na SEAPROF e adaptados pelos autores.

seca seja carbono, como revelam Silveira et al. (2008).

E, por sugestão de Cotta et al. (2006), os quais citam que convertendo-se o carbono em $\mathrm{CO}_{2}$ eq. obtém-se os RCEs, multiplicou-se o total de carbono por 44/12 (razão do peso molecular do $\mathrm{CO}_{2}$ e do carbono, respectivamente). Assim, 3,67 $\mathrm{tCO}_{2}$ eq. correspondem a uma tonelada de carbono. e) Custo de transação no âmbito do MDL

Para quantificar o custo de transação no âmbito do MDL, fixou-se o sequestro nos plantios em estudo em $16.000 \mathrm{tCO}_{2}$ eq./ano (ou $480.000 \mathrm{tCO}_{2}$ eq. em 30 anos). Adotou-se tal procedimento pois esse é o valor máximo, segundo UNFCCC (2003), para que o projeto de MDL 
Florestal seja classificado como de pequena escala. Segundo Lima (2010), um reflorestamento de Hevea spp. no Acre, ao final do seu ciclo de 30 anos, sequestra $25,87 \mathrm{t} /$ ha de carbono, o qual, convertido para $\mathrm{CO}_{2}$ eq. (multiplicando-se por 3,67), equivale a 94,94 RCEs.

Assim sendo, dividindo-se $480.000 \mathrm{tCO}_{2}$ eq. por 94,94 tCO $_{2}$ eq., identificou-se a área de 5.055,83 ha como necessária para a absorção de 16.000 tCO eq./ano.

Complementando, considerou-se, por indicação de Cotta et al. (2006), o valor de US\$ 260.000 como custo fixo do projeto. E, dividindo-se esse valor pela área de plantio considerada nesta análise (5.055,83 ha), obteve-se o custo de US\$51,42/ha, o qual ocorre no ano de implantação do projeto (ano zero).

Salienta-se que Mastrângelo (2008) afirma que, devido ao complexo e demorado trâmite dos projetos de MDL desde sua pré-concepção até a emissão e negociação das RCEs, eles apresentam altos custos de transação.

f) Preço da RCEs no Mercado de Carbono para avaliação de projetos

Foi adotado, como indicam J. Malucelli (2010), o preço da tonelada de $\mathrm{CO}_{2}$ eq. (RCEs) de 10 euros ou US\$12,85.

É oportuno citar que os dados usados neste estudo foram corrigidos segundo o Índice Geral de Preços do Mercado - IGP-M para janeiro de 2012. Feito isso, esses valores foram convertido de reais $(\mathrm{R} \$$ ) para dólares (US\$), considerando-se a cotação oficial de venda do dólar de janeiro/2012, dada pelo Banco Central do Brasil (2012).

\subsection{Métodos}

\subsubsection{Receitas de geração das Reduções Certificadas de Emissões (RCEs)}

Neste estudo, a proposta da geração de RCEs baseou-se nas premissas básicas já definidas nas COPs para aprovar projetos MDL. Esses RCEs são, então, contabilizados em função do volume gerado pelo povoamento de Hevea spp. no ciclo de 30 anos.

A receita das Reduções Certificadas de Emissões (RCEs) considerada neste estudo foi de US\$1.219,98/ha. Esse valor foi obtido multiplicando-se o preço da tonelada de $\mathrm{CO}_{2}$ eq. (RECEs) de US\$12,85 por $94,94 \mathrm{RCEs} /$ ha.

\subsubsection{Taxa Mínima de Atratividade (TMA)}

Seguindo sugestão de Cotta et al. (2006) e Valdetaro et al. (2011), neste estudo foi empregada a taxa de desconto indicada por Brasil (2002), de 10\%.

\subsubsection{Análise econômica}

De acordo com o recomendado por Berger et al. (2011), fez-se uso, nesta análise econômica, das seguintes ferramentas para a avaliação de projetos florestais: Valor Presente Líquido (VPL), Taxa Interna de Retorno (TIR) e Valor Esperado da Terra (VET).

a) Valor Presente Líquido - VPL

No cálculo do Valor Presente Líquido, como propõem Berger et al. (2011), foi adotada a seguinte expressão matemática:

$V P L=\sum_{j=0}^{n} R_{j}(1+i)^{-j}-\sum_{j=0}^{n} C_{j}(1+i)^{-j}$

em que: Rt é a receita no período de tempo j considerado; Ct representa os custos no período de tempo j considerado; n é a duração do projeto em anos ou em número de períodos; e i é a taxa de juros adotada, expressa de forma decimal.

b) Taxa Interna de Retorno - TIR

Para a identificação da Taxa Interna de Retorno, seguindo sugestões de Cotta et al. (2006), fez-se uso da fórmula apresentada a seguir:

$\sum_{j=0}^{n} R_{j}(1+i)^{-j}=\sum_{j=0}^{n} C_{j}(1+i)^{-j}$

em que: $\mathrm{R}_{\mathrm{j}}$ são as receitas no ano $\mathrm{j}$; $\mathrm{C}_{\mathrm{j}}$ refere-se ao custo no ano j; e n é a duração do projeto, em anos.

c) Valor Esperado da Terra - VET

Por indicação de Silva \& Fontes (2005), foi empregada a seguinte expressão para o cálculo do Valor Esperado da Terra:

$V E T=\frac{V_{0} R L(1+i)^{t}}{(1+i)^{t}-1}$

em que: $V_{0} R L$ representa o valor atual da receita líquida que se repete a cada ciclo. 
d) Análise de sensibilidade

Com base nos procedimentos usados por Cotta et al. (2006), fez-se uma análise de sensibilidade, na qual foram avaliados os resultados gerados nos critérios VPL e TIR após, mantidos os custos constantes, uma variação de $20 \%$ para mais ou para menos na renda básica dos projetos estudados (renda com as RCEs e a venda do látex, apurada no levantamento para a realização deste estudo).

Como uma segunda análise de sensibilidade realizada nesta pesquisa foi identificado o impacto, nos resultados gerados de VPL e da TIR dos plantios de seringueira em questão, de se considerar, ou não, o custo do fator terra nesses projetos.

\section{RESULTADOS E DISCUSSÃO}

O processamento dos dados usados neste artigo geraram os seguintes resultados.

\subsection{Fluxo de caixa}

A Tabela 2 apresenta o fluxo de caixa do plantio no âmbito do MDL Florestal com inclusão dos custos de transação de projetos de MDL e receitas da venda de RCEs.

\subsection{Análise econômica}

Os resultados obtidos para o cálculo de VPL, TIR e VET para os seringais de cultivo no âmbito do MDL implantados no Acre são apresentados e discutidos a seguir.

\subsubsection{Valor Presente Líquido - VPL}

Na Tabela 3 tem-se o comportamento do Valor Presente Líquido (VPL) do projeto de reflorestamento de Hevea spp., no âmbito do MDL Florestal

Os valores da Tabela 3 revelam que, de maneira geral, os reflorestamentos com Hevea spp. implantados no Acre com o intuito de se beneficiarem do mercado de carbono gerarão lucro. A única exceção é que, considerando o custo do fator terra, esses projetos não seriam viáveis caso a sua renda sofra diminuição de $20 \%$ em relação ao valor básico da receita obtido neste estudo.
Salienta-se que, no seu estudo abordando plantios de seringueira no estado de São Paulo, Nishi et al. (2005) encontraram um VPL de US\$ 963,84/ha.

\subsubsection{Taxa Interna de Retorno - TIR}

A Tabela 4, por sua vez, sintetiza a evolução da TIR nos projetos de reflorestamento de Hevea spp. no âmbito do MDL Florestal analisados neste estudo.

Verifica-se, nos números da Tabela 4, que os seis cenários avaliados neste estudo geraram um valor médio da Taxa Interna de Retorno de 13,32\% ao ano. Com uma ressalva: na situação em que é considerado o custo do fator terra, tem-se como sua renda um valor reduzido em $20 \%$ da receita básica encontrada neste estudo - a TIR calculada tem um valor menor que a taxa de juro adotada nesta análise.

É oportuno destacar que Cotta et al. (2006) obtiveram um valor da taxa interna de retorno de $24,12 \%$ a.a. para um consórcio de seringueira e cacau. Já Nishi et al. (2005) chegaram a uma TIR de 15,56\% a.a. no seu estudo avaliando plantios de seringueira no estado de São Paulo, enquanto Valdetaro et al. (2011), analisando projetos de reflorestamento com seringueira na Bahia, obteve uma taxa interna de retorno de $71 \%$ ao ano.

Complementando, observa-se que Mastrângelo (2008) chegou a uma TIR igual a 13,05\% a.a., válida para povoamentos com Teca (Tectona grandis L.f.), implantados no Acre, com o objetivo de também se inserir no mercado de carbono.

\subsubsection{Valor Esperado da Terra - VET}

A Tabela 5 mostra o comportamento do Valor Esperado da Terra em projetos de reflorestamento de Hevea spp. no âmbito do MDL.

Observando-se os resultados apresentados na Tabela 5, constata-se que em apenas uma das três situações avaliadas os reflorestamentos com Hevea spp. não seriam possíveis de serem implantados no estado do Acres. Mais especificamente, o caso no qual se pressupõe uma queda na receita do projeto em $20 \%$ em relação ao valor da renda média obtido neste estudo. Nesse cenário, o valor máximo que se poderia pagar pela terra, para que esses empreendimentos fossem remunerados na taxa de $10 \%$ a.a. (expresso pelo VET calculado), é menor que o preço médio da terra própria para reflorestamento praticado no estado do Acre (US\$ 615, 91/ha). 
Tabela 2. Fluxo de caixa dos plantios de Hevea spp. inseridos no MDL Florestal, estado do Acre, 2012.

Table 2. Cash flow of Hevea spp. reforestation for Forestry CDM, state of Acre, 2012.

\begin{tabular}{|c|c|c|c|c|}
\hline Ano & Atividade & Custos (US\$) & Produção (kg/ha) & Receitas (US\$) \\
\hline 0 & Implantação & $4.224,65$ & 0,00 & \\
\hline 1 & Manutenção & 554,84 & 0,00 & $1.219,98$ \\
\hline 2 & Manutenção & 567,74 & 0,00 & \\
\hline 3 & Manutenção & 566,13 & 0,00 & \\
\hline 4 & Manutenção & 537,63 & 0,00 & \\
\hline 5 & Manutenção & 306,45 & 0,00 & \\
\hline 6 & Manutenção & 683,33 & 0,00 & \\
\hline 7 & Manutenção & $1.069,89$ & 600,00 & $1.128,00$ \\
\hline 8 & Produção & $1.080,65$ & 800,00 & $1.504,00$ \\
\hline 9 & Produção & $1.091,40$ & 950,00 & $1.786,00$ \\
\hline 10 & Produção & $1.091,40$ & $1.100,00$ & $2.068,00$ \\
\hline 11 & Produção & $1.091,40$ & $1.300,00$ & $2.444,00$ \\
\hline 12 & Produção & $1.091,40$ & $1.350,00$ & $2.538,00$ \\
\hline 13 & Produção & $1.091,40$ & $1.500,00$ & $2.820,00$ \\
\hline 14 & Produção & $1.091,40$ & $1.700,00$ & $3.196,00$ \\
\hline 15 & Produção & $1.091,40$ & $2.000,00$ & $3.760,00$ \\
\hline 16 & Produção & $1.091,40$ & $2.000,00$ & $3.760,00$ \\
\hline 17 & Produção & $1.091,40$ & $2.000,00$ & $3.760,00$ \\
\hline 18 & Produção & $1.091,40$ & $2.000,00$ & $3.760,00$ \\
\hline 19 & Produção & $1.091,40$ & $2.000,00$ & $3.760,00$ \\
\hline 20 & Produção & $1.091,40$ & $2.000,00$ & $3.760,00$ \\
\hline 21 & Produção & $1.091,40$ & $2.000,00$ & $3.760,00$ \\
\hline 22 & Produção & $1.091,40$ & $2.000,00$ & $3.760,00$ \\
\hline 23 & Produção & $1.091,40$ & $2.000,00$ & $3.760,00$ \\
\hline 24 & Produção & $1.091,40$ & $2.000,00$ & $3.760,00$ \\
\hline 25 & Produção & $1.091,40$ & $2.000,00$ & $3.760,00$ \\
\hline 26 & Produção & $1.091,40$ & $2.000,00$ & $3.760,00$ \\
\hline 27 & Produção & $1.091,40$ & $2.000,00$ & $3.760,00$ \\
\hline 28 & Produção & $1.091,40$ & $2.000,00$ & $3.760,00$ \\
\hline 29 & Produção & $1.091,40$ & $2.000,00$ & $3.760,00$ \\
\hline 30 & $\begin{array}{c}\text { Produção + } \\
\text { Revenda da terra/ }\end{array}$ & $1.091,40$ & $2.000,00$ & $4.478,56$ \\
\hline
\end{tabular}

Fonte: elaborado pelos autores.

Tabela 3. Valor Presente Líquido em plantios de Hevea spp. inseridos no MDL Florestal, estado do Acre, 2012.

Table 3. Net Present Value of Hevea spp. reforestation for forestry CDM projects, state of Acre, 2012.

\begin{tabular}{lccc}
\cline { 2 - 4 } \multicolumn{1}{c}{ Critério terra } & \multicolumn{3}{c}{ Critério preço } \\
\cline { 2 - 4 } & $\begin{array}{c}\text { Renda do projeto 20\% } \\
\text { menor que o valor básico }\end{array}$ & Renda básica do projeto & $\begin{array}{c}\text { Renda do projeto 20\% } \\
\text { maior que o valor básico }\end{array}$ \\
\hline Considerando a terra & $-34,37$ & $2.969,71$ & $5.973,78$ \\
\hline Não considerando a terra & 546,25 & $3.550,32$ & $6.554,40$ \\
\hline
\end{tabular}

Tabela 4. Taxa Interna de Retorno dos plantios de Hevea spp. no MDL Florestal, estado do Acre, 2012.

Table 4. Internal Rate o Return of Hevea spp. reforestation for forestry CDM projects, state of Acre, 2012.

\begin{tabular}{lccc}
\cline { 2 - 3 } \multicolumn{1}{c}{ Critério terra } & \multicolumn{3}{c}{ Critério preço } \\
\cline { 2 - 4 } & $\begin{array}{c}\text { Renda do projeto 20\% } \\
\text { menor que o valor básico }\end{array}$ & Renda básica do projeto & $\begin{array}{c}\text { Renda do projeto 20\% } \\
\text { maior que o valor básico }\end{array}$ \\
\hline Considerando a terra & 9,95 & 13,04 & 15,62 \\
Não considerando a terra & 10,68 & 13,93 & 16,67 \\
\hline
\end{tabular}


Tabela 5. Valor Esperado da Terra em projetos de reflorestamento de Hevea spp. no âmbito do MDL Florestal, 2012. Table 5. Land Expectation Value in reforestation projects of Hevea spp. for forestry CDM projects, 2012.

\begin{tabular}{ccc}
\hline & Critério renda do projeto & \\
\hline $\begin{array}{c}\text { Renda do projeto } \mathbf{2 0} \% \\
\text { menor que o valor básico }\end{array}$ & Renda básica do projeto & $\begin{array}{c}\text { Renda do projeto } \mathbf{2 0} \% \\
\text { maior que o valor básico }\end{array}$ \\
\hline 579,45 & $3.766,15$ & $6.952,85$ \\
\hline
\end{tabular}

Cabe aqui mencionar que, pesquisando plantios com seringueiras no estado de São Paulo, Nishi et al. (2005) chegaram a um valor esperado da terra de US\$1.022,43.

\section{CONCLUSÕES}

- Os reflorestamentos com seringueira destinados ao sequestro de carbono no âmbito do MDL Florestal implantados no estado do Acre apresentam potencial para serem viáveis e, assim, fortalecerem a economia de base florestal dessa região.

- O limitante para que, no Acre, plantios com Hevea spp. visando o sequestro de carbono, no âmbito do MDL Florestal, sejam viáveis, economicamente, é que sua renda não sofra queda de $20 \%$, ou mais, em relação ao valor médio obtido neste estudo.

\section{STATUS DA SUBMISSÃO}

Recebido: 20 out., 2013

Aceito: 01 mar., 2016

\section{AUTOR(ES) PARA CORRESPONDÊNCIA}

\section{Aldione da Silva Lessa}

Serviço Brasileiro de Apoio às Micro e Pequenas Empresas - SEBRAE-Acre, Rua Rio Grande do Sul, 109, CEP 69900-092, Rio Branco, AC, Brasil e-mail: aldionelessa@gmail.com

\section{REFERÊNCIAS}

Acre. Governo do Estado. Acre em números. Acre: Portal do Governo do Acre; 2011. [online]. [citado em 2011 ago. 9]. Disponível em: http://www.ac.gov.br

Banco Central do Brasil. Taxa de câmbio. Brasília: Banco Central do Brasil; 2012. [online]. [citado em 2012 jan. 20]. Disponível em: http://www4.bcb.gov.br/pec/taxas/ port/ptaxnpesq.asp?id=txcotacao
Berger R, Santos AJ, Timofeiczyk R Jr, Bittencourt AM, Souza VS, Eisfeld CL. Efeito do custo da terra na rentabilidade florestal: Um estudo de caso para Santa Catarina. Revista Floresta 2011; 41(3): 599-610.

Brasil. Ministério da Ciência, Tecnologia e Inovação MCTI. Diversos documentos [online]. Brasília: MCTI; 2012. [citado em 2012 jan. 20] Disponível em: http:// www.mct.gov.br

Brasil. Ministério do Meio Ambiente - MMA. Critérios e indicadores de elegibilidade para avaliação de projetos candidatos ao mecanismo de Desenvolvimento limpo (MDL) [online]. Brasília: MMA; 2002. [citado em 2002 abr. 24]. Disponível em: http://www.centroclima.coppe. ufrj.br/new2/ccpdf/criterio.pdf

Centro de Gestão e Estudos Estratégicos: Ciência, Tencologia e Inovação - CGEE. Manual de capacitação sobre mudança climática e projetos de mecanismo de desenvolvimento limpo ( $M D L)$. Brasília: Centro de Gestão e Estudos Estratégicos; 2010.

Cotta MK, Jacovine LAG, Paiva HN, Soares CPB, Virgens AC. Fo, Valverde S. Análise econômica do consórcio seringueira-cacau para geração de certificados de emissões reduzidas. Revista Árvore 2006; 30(6): 969-979. http:// dx.doi.org/10.1590/S0100-67622006000600012.

Cotta MK, Jacovine LAG, Paiva HN, Soares CPB, Virgens AC Fo, Valverde SR. Quantificação de biomassa e geração de certificados de emissões reduzidas no consórcio seringueira-cacau. Revista Árvore 2008; 32(6): 969-978. http://dx.doi.org/10.1590/S0100-67622008000600002.

Fernandes, TJG. Contribuição dos certificados de emissões reduzidas (CERs) na viabilidade econômica da heveicultura [dissertação]. Viçosa: Universidade Federal de Viçosa; 2003.

Forest Trends, The Katoomba Group. Aprendendo sobre Serviços Ambientais (FT e TKG): manual de orientação para o desenvolvimento dos subprogramas do Sistema de Incentivos a Serviços. [online]; 2011. [citado em 2012 abr 6]. Disponível em: http://www.forest-trends.org/ documents/files/doc_2542.pdf

Instituto Brasileiro de Geografia e Estatística - IBGE. Censo 2010 [online]. Rio de Janeiro: IBGE; 2010 [citado em 2013 maio 10]. Disponível em: http://www.ibge.gov. br/estadosat/perfil.php?sigla $=\mathrm{ac}$

Intergovernmental Panel on Climate Change - IPCC. Quarto relatório de avaliação do painel intergovernamental sobre mudança do clima [online]; 2007. [citado em 2007 
dez 1]. Disponível em: http://www.ccst.inpe.br/Arquivos/ ipcc_2007.pdf

J. Malucelli, CMC Ambiental. Carbon finance activities. Curitiba; 2010.

Lima LCC. Quantificação de Biomassa em um plantio de Hevea spp. (seringueira) no município de Bujari, Acre [monografia]. Rio Branco: Centreuo de Ciências Biológicas e da Natureza, Universidade Federal do Acre; 2010.

Mastrângelo JPS. Mudanças Climáticas e o Protocolo de Quioto: Implicações para a inserção da Região Amazônica no Mercado de Carbono [dissertação]. Rio Branco: Programa de Pós-graduação Mestrado em Desenvolvimento Regional, Universidade Federal do Acre; 2008.

Nishi MH. O MDL e o atendimento aos critérios de elegibilidade e indicadores de sustentabilidade por diferentes atividades florestais [dissertação]. Viçosa: Universidade Federal de Viçosa; 2003.

Nishi MH, Jacovine LAG, Silva ML, Valverde SR, Nogueira HPN, Alvarenga AP. Influência dos créditos de carbono na viabilidade financeira de três projetos florestais. Revista Árvore 2005; 29(2): 263-270. http://dx.doi.org/10.1590/ S0100-67622005000200009.

Sanguino AC, Santana AC, Homma AKO, Barros PLC, Kato OK, Amin MMGH. Análise econômica de investimentos em Sistemas de Produção Agroflorestal no Estado do Pará. Revista de Ciências Agrárias 2007; 47(1): 23-47.

Silva ML, Fontes AA. Discussão sobre os critérios de avaliação econômica: Valor Presente Líquido (VPL), Valor Anual Equivalente (VAE) e Valor Esperado da Terra (VET). Revista Árvore 2005; 29(6): 931-936. http://dx.doi. org/10.1590/S0100-67622005000600012.

Silveira P, Koehler SH, Sanquetta CR, Arce JE. O estado da arte na estimativa de biomassa e carbono em formações florestais. Revista Floresta 2008; 38(1): 186-206.

Stern N. The economics of climate change: the stern review. Cambridge: University of Cambridge; 2006.

United Nations Framework Convention on Climate Change - UNFCCC. The convention and Kyoto Protocol [online]; 2003. [citado em 2011 dez. 1]. Disponível em: http://unfccc.int/resource/convkp.html

Valdetaro EB, Silva FL, Ribeiro SC, Jacovine LAG. Contribuição dos créditos de carbono na viabilidade econômica dos contratos de fomento florestal no sul da Bahia. Revista Árvore 2011; 35(6): 1307-1317. http:// dx.doi.org/10.1590/S0100-67622011000700017.

Walter MKC, Coltri PP. Geração de créditos de carbono por sistemas florestais. Revista da Madeira 2009; 20(120): 32-36. 